\title{
Expression of Bt Gene (cry1Ac) on Different Plant Parts at Different Stages in Bt Cotton Genotypes
}

\author{
B.H. Nagappa* and B.M. Khadi \\ Agricultural Research Station (UAS, Dharwad), Hanumanamatti, Ranebennur Taluk, \\ Haveri District, India \\ *Corresponding author
}

\section{A B S T R A C T}

\section{Keywords}

Bt Gene $($ crylAc), Bt Cotton

Genotypes, Gene expression

Article Info

Accepted:

26 December 2017

Available Online:

10 January 2018
Cotton yield losses due to insect pests are estimated to be around 10 to 14 per cent every year. These insect protected cotton varieties contain a naturally occurring substance, Bacillus thuringiensis (Bt) protein which has been used as an ingredient in safe and effective biological sprays for more than 50 years. Of the total available Bt cotton hybrids in 2006, four different events containing Cry1Ac gene (MON 531 event) by Mahyco sourced from Monsanto, stacked Cry X (Cry1 Ac and Cry2Ab) gene event (MON 15985) developed by Mahyco sourced from Monsanto, Cry1Ac gene (Event 1) by JK-seeds sourced from IIT Kharagpur and fusion gene Cry1Ab and Cry1Ac (GF Mervent) by Nath seeds sourced from China have received commercial approval for sale in Northern, Central and Southern cotton growing zones. The expression of Bt gene varies with different parts of plant. The Cry 1 Ac gene expression highest in leaf $(4.31 \mu \mathrm{g} / \mathrm{g}$ at $45 \mathrm{DAS})$ followed by young boll $(2.02 \mu \mathrm{g} / \mathrm{g}$ at $105 \mathrm{DAS})$, flower $(>1.9 \mu \mathrm{g} / \mathrm{g} 105 \mathrm{DAS})$, seeds $(1.9 \mu \mathrm{g} / \mathrm{g} 120$ DAS and $1.49 \mu \mathrm{g} / \mathrm{g}$ at 160 DAS and least in rind $(0.63 \mu \mathrm{g} / \mathrm{g} 105 \mathrm{DAS})$.

\section{Introduction}

Cotton has a proud place among the cash crops from the earliest times. It finds mention in the Rigveda the oldest scripture of the Hindus. Manu, the law giver also referred to it in his Dharma Shastra. It was the excellence of Indian cotton fibres famed as webs of woven wind which compelled European countries to seek new trade routes with India. Even though, nowadays advent of a multitude of other fibres, cotton; white gold rules the world of textile. So, it is unchallenged as a natural textile fibre. It is an important fibre and food crop of nearly 100 countries with China, India, United States, Pakistan and Brazil being five of the largest producers of cotton.

In India, yields were low compared to world average partly because of heavy losses from insect pests particularly cotton bollworm complex. Yield losses due to insect pests are estimated to be around 10 to 14 per cent every year. So, India is addressing the need for increased Bt cotton cultivars. These insect 
protected cotton varieties contain a naturally occurring substance, Bacillus thuringiensis (Bt) protein which has been used as an ingredient in safe and effective biological sprays for more than 50 years. Bt trait has been successfully transferred into several Indian lines. Extensive and fully replicated field trials of Bt cotton were conducted from 1998 to 2001 cropping seasons, meeting the government requirements for commercialization. Three Bt cotton cultivars have been approved for planting in India in 2002-03. Since, the introduction of Bt cotton hybrid around 44,500ha were planted with three hybrids of Bt cotton in central and southern zones in 2002-03 season. This increased to some 1, 00,000 ha in 2003-04. In 2004-05 around four Bt cotton hybrids were planted over $5,00,000$ ha by three lakh resource poor farmers. With approval of 16 new hybrids of half a dozen companies including six Bt cotton hybrids for northern region, Bt cotton planting for 2005-06 season has experienced the highest yearly percentage growth rate increasing its area by 160 per cent (13 lakh ha). Presently India has the fourth largest area $(11.57 \mathrm{mh})$ after Argentine, Brazil and USA under genetically modified Bt cotton crops, according to the International service for the Acquisition of Agri Biotech Application (ISAAA). Area under Bt cotton hybrids in northern, central and southern cotton growing zones of India increased upto 96 percent in 2014 and production was 40 million bales from 13 million bales during the year 2002 .

In a landmark decision, the Genetic Engineering Approval Committee (GEAC) of the Ministry of Environment and Forest (MOEF) has approved 49 new hybrids of $\mathrm{Bt}$ cotton varieties to sale this year in addition to the $62 \mathrm{Bt}$ cotton hybrids approved until 2006. This brings the total of Bt cotton hybrids to 111 (49 approvals) giving farmers of India's three cotton growing zones more choices about which varieties to cultivate. This includes 14 hybrids containing three events to be sold by thirteen companies in Southern Zone of India. Of the total available Bt cotton hybrids in 2006, four different events containing Cry1 Ac gene (MON 531 event) by Mahyco sourced from Monsanto, stacked Cry $\mathrm{X}$ (Cry1Ac and Cry2Ab) gene event (MON 15985) developed by Mahyco sourced from Monsanto, Cry1Ac gene (Event 1) by JKseeds sourced from IIT Kharagpur and fusion gene Cry1 Ab and Cry1Ac (GF Mervent) by Nath seeds sourced from China have received commercial approval for sale in Northern, Central and Southern cotton growing zones. Till now there is not much information regarding expression of Bt gene in different genotypes at different stages of crop life and different parts of the plant.

\section{Materials and Methods}

There are 36 hybrids Bt hybrids viz.,JKCH224,SDCH-302, K-5308, KDCH-441， JK Indra, RCH-2,JK Ishwar, JKCH-1947, RCH134, JKCH-22, Ankur-651 BGI, Ankur-651 BGI, RCH-144, JKCH-1050, SBCH311,PCH-2270,NECH-3R, KDCHH-9810, NECN-2R, RCH-118, JKCH-226, Dhruva, K5316, KDCHH-9632, JK Varun, RCH-2171, RCH-20, JK Durga, JKCH-99, RCH-138, JKCH-1945, JK Gowri, RCH-377, VCH-111, NHH-44, VICH-5, VICH-5 and VICH-9 have collected for expression of Cry $1 \mathrm{Ac}$ protein in different parts of plant at different growth stages of Bt cotton.

Samples of squares, flower, boll, seed and leaf were used for extracting the toxin. Desi Gen Quan-T ELISA 96 well plate kits supplied by Desi Gen Mahyco, Company Jalna, Maharashtra, India were used to estimate the toxin content in the plant parts. Distilled water, Powder A, Powder B, 10x buffer, 1x buffer, Extraction buffer, Cry1Ac stock solution, AP conjugated $\mathrm{Ab}$ were supplied by 
the said company. The samples viz., squares flower, bolls seed and leaf were collected in ice box and carried to laboratory for further analysis as per protocol provided with quantification kit.

\section{$\delta$-endotoxin quantification protocol}

Twenty mg of sample from each genotype was weighed and placed in $1.5 \mathrm{ml}$ microfuge tube for further analysis. $500 \mu \mathrm{l}$ of ice-cold $1 \times$ sample extraction buffer was added (add $0.2 \mathrm{~g}$ powder $\mathrm{A}$ and $12 \mathrm{~g}$ powder $\mathrm{B}$ to $100 \mathrm{ml}$ sample extraction buffer prepared freshly at the time of sample extraction).

Samples were macerated manually and contents were chilled on ice for $10 \mathrm{~min}$ and again macerated for $30 \mathrm{sec}$. The contents were spun at $8000 \mathrm{rpm}$ in a micro centrifuge for 15 min and supernatant was pipetted out. Pipetted supernatant was diluted to $1: 4$ proportion using diluent buffer (diluent buffer : Add 100 $\mathrm{ml}$ of $10 \mathrm{X}$ buffer A1, dilute it to one litre by using deionized water added with $0.5 \%$ ovalbumin in $1 \mathrm{X}$ buffer).

Preparation of positive and negative QC seed extract

$500 \mu 11 \mathrm{X}$ buffer A was added to the positive and negative seed samples provided with the kit. It was crushed well with a disposable plastic pestle and spun for $30 \mathrm{sec}$ in a micro centrifuge at $2000 \mathrm{rpm}$ and $100 \mu \mathrm{l}$ of each supernatant per well was used.

\section{Standard curve generation}

$20 \mathrm{ng}$ per $\mathrm{ml}$ working stock solution was prepared from $20 \mu \mathrm{l}$ per ml CrylAc stock solution provided in 1X diluent buffer (add 20 $\mu 1$ Cry1Ac stock $+980 \mu 11 \mathrm{X}$ diluent buffer). Other quantification standards were prepared as under.

\section{Results and Discussion}

With cotton attracting a wide range of pests and the attendant high pesticide usage leading to a polluted atmosphere and increased cost of cultivation, a rethink was possible in the form of environment friendly pest biocontrol procedures. The introduction of $\mathrm{Bt}$ genes (Cry1Ac) against bollworms into cotton has revitalized cotton cultivation and made it profitable again. In the present study, the expression pattern of the Cry1Ac gene was investigated so as to pave the way for further studies relating it to insect control and efficiency.

Several Bt hybrids have been released in India and any Bt hybrid which produced Bt toxin above the critical level in different parts of plant and later stages of crop life span was very important and formed a part of the present investigation. The expression level of Cry1Ac endotoxin protein was estimated in leaves, flower, square, boll, rind and seed in $36 \mathrm{Bt}$ hybrids at different stages of crop growth and results are presented in Table 1, 2, 3,4 and 5.

\section{Quantitative estimation of Cry1Ac endotoxin in leaf}

Dong and Lis (2007) reported variability in Bt cotton efficacy in particular on induced variability by environmental stresses. Insecticidal protein content in $\mathrm{Bt}$ cotton is variable with plant age, plant structure or under certain environmental stresses. Reduction of Bt protein content in late season cotton could be due to the over expression of Bt gene at earlier stages which leads to gene regulation at post transcription levels and consequently results in gene silencing at later stages. Methylation of promoter may be also involved in the declined expression of endotoxin proteins. Kranthi et al., (2005) also reported that Cry1Ac expression was variable 
among the hybrids and also between different plant parts. The leaves of $\mathrm{Bt}$ cotton plants were found to have the highest levels of Cry1Ac expression followed by young boll, bolls rind and then in flowers. The toxin expression in the bolls rind, square bud and ovary of flower was clearly inadequate to confer full protection to fruiting parts. There was a correlation between Cry1Ac expression in Bt cotton and $H$. armigera mortality thus identifying a critical expression level for Cry1Ac at $1.9 \mu \mathrm{g}$ per $\mathrm{g}$ in tissue, below which Helicoverpa armigera would be able to survive.

Level of Cry1Ac Bt protein $(\mu \mathrm{g} / \mathrm{g})$ in leaf at different stages of crop growth of Bt cotton hybrids have been presented in Table 1 and Figure 1.

Cry1Ac Bt protein test started from 45 days after sowing. In the leaf, on an average Cry1Ac expression at 45 DAS was $4.21 \mu \mathrm{g}$ per $g$ and it declined to $1.83 \mu \mathrm{g}$ per gram at 90 DAS. The decline in Cry1Ac protein was 56.54 per cent. From 90 to 120 DAS decline of Cry1Ac was 60.11 per cent. Mean Cry1Ac level of 36 hybrids at 120 DAS was $0.73 \mu \mathrm{g}$ per g. Average protein expression was $0.60 \mu \mathrm{g}$ per $\mathrm{g}$ of leaf tissue at 140 DAS and revealed a 17.82 per cent decrease from 120 to 140 DAS. At $160 \mathrm{DAS}, 0.33 \mu \mathrm{g}$ per $\mathrm{g} \mathrm{Bt}$ protein was observed. The per cent of decline of Cry1Ac from 140 to 160 DAS was 45 per cent in the leaf.

A total of 36 hybrids were tested for expression of Cry1Ac Bt protein, which showed more than critical level $(1.9 \mu \mathrm{g} / \mathrm{g})$ of mortality at 45 DAS in leaf tissue. However, hybrids JK-Gowri (0.03 $\mu \mathrm{g} / \mathrm{g})$, JKCH-1050 $(0.07 \mu \mathrm{g} / \mathrm{g})$ and JKCH-266 $(0.08 \mu \mathrm{g} / \mathrm{g})$ were exceptions to the above statement. At 90 DAS, hybrids JKCH-1050 (0.09 $\mu \mathrm{g} / \mathrm{g})$, SBCH-311 $(1.10 \mu \mathrm{g} / \mathrm{g}), \mathrm{PCH}-2270$ (1.62 $\mu \mathrm{g} / \mathrm{g}), \mathrm{NCEN}-2 \mathrm{R}$ $(0.03 \mu \mathrm{g} / \mathrm{g}), \mathrm{RCH}-118(1.73 \mu \mathrm{g} / \mathrm{g}), \mathrm{JKCH}-266$
(0.08 $\mu \mathrm{g} / \mathrm{g}), \mathrm{K}-5316(1.04 \mu \mathrm{g} / \mathrm{g}), \mathrm{JK}-$ Varun $(0.28 \mu \mathrm{g} / \mathrm{g}), \mathrm{JK}-$ Gowri $(0.09 \mu \mathrm{g} / \mathrm{g})$, VICH-111 $(1.01 \mu \mathrm{g} / \mathrm{g}), \mathrm{VICH}-5(0.62 \mu \mathrm{g} / \mathrm{g})$ and VICH-9 $(1.68 \mu \mathrm{g} / \mathrm{g})$ had Cry1Ac protein production significantly less than critical level (1.90 $\mu \mathrm{g} / \mathrm{g}$ ). But rest of the hybrids (24) exhibited Cry1Ac production significantly higher than critical level $(1.90 \mu \mathrm{g} / \mathrm{g})$ of mortality. At 120 , 140 and 160 DAS Cry1Ac expression level of all 36 hybrids was significantly less than critical level $(1.90 \mu \mathrm{g} / \mathrm{g})$. The Cry1Ac expression showed a declining trend from 45 to 160 DAS. Similar results were observed by Kranthi et al., (2005) and Dong and Lis (2007).

The endotoxin (Cry1Ac) content in boll rind at different stages of crop growth is given in Table 2 and Figure 2.

In the rind, Cry1Ac expression at 105, 120, 140 and 160 DAS was $0.63,0.53,0.43$ and $0.23 \mu \mathrm{g}$ per $\mathrm{g}$, respectively. The per cent of Cry1Ac decline from 105 to 120,120 to 140 and 140 to 160 DAS was $15.88,18.87$ and 46.51 per cent, respectively. None of the Bt hybrids recorded Cry1Ac protein significantly higher than critical level in the rind. Kranthiet al., (2005) noticed 0.25 to $0.37 \mu \mathrm{g}$ per $\mathrm{g}$ Cry1Ac protein in rind.

Endotoxin levels quantified in seeds at different stages of crop growth is given in Table 3 and Figure 3.

The expression of Cry1Ac at 105, 120, 140 and 160 DAS was $0.73,1.90,0.64$ and $1.49 \mu \mathrm{g}$ per $\mathrm{g}$, respectively in seed tissue. The increase of Cry1Ac from 105 to 120 DAS was 160 per cent. From 120 to 140 DAS, decrease of Cry1Ac protein was 66.31 per cent. The increase of Cry1Ac from 140 to 160 DAS was 132.8 per cent. At 105 and 140 DAS, none of the Bt hybrids had higher than $1.90 \mu \mathrm{g}$ per $\mathrm{g}$ of Cry1Ac protein in seeds. Like in leaf and rind, the levels of CrylAc in seed also 
decreased from 120 to 140 DAS $(0.64 \mu \mathrm{g} / \mathrm{g})$ but again Cry 1 Ac protein production increase from 140 DAS to160 DAS by 132.8 per cent.

The Bt hybrids viz., JKCH-2245 (1.87 $\mu \mathrm{g} / \mathrm{g})$, SBCH-302 (2.33 $\mu \mathrm{g} / \mathrm{g})$, K-5038 (2.58 $\mu \mathrm{g} / \mathrm{g})$, JKCH-1947 (3.22 $\mu \mathrm{g} / \mathrm{g}), \quad \mathrm{JKCH}-22 \quad(3.35$ $\mu \mathrm{g} / \mathrm{g}), \mathrm{RCH}-144$ (1.84 $\mu \mathrm{g} / \mathrm{g}), \mathrm{SBCH}-311$ (4.15 $\mu \mathrm{g} / \mathrm{g}), \mathrm{PCH}-2270(2.90 \mu \mathrm{g} / \mathrm{g}), \mathrm{KDCHH}-9810$ (1.92 $\mu \mathrm{g} / \mathrm{g}), \mathrm{RCH}-118(1.18 \mu \mathrm{g} / \mathrm{g})$, Dhruva (4.17 $\mu \mathrm{g} / \mathrm{g}), \mathrm{K}-5316(2.20 \mu \mathrm{g} / \mathrm{g})$, KDCHH$9632(2.28 \mu \mathrm{g} / \mathrm{g})$, JK-Varun (2.33 $\mu \mathrm{g} / \mathrm{g})$, RCH-20 (1.87 $\mu \mathrm{g} / \mathrm{g})$, JK-Durga $(3.01 \mu \mathrm{g} / \mathrm{g})$, JKCH-1945 (2.11 $\mu \mathrm{g} / \mathrm{g}), \quad \mathrm{VICH}-111 \quad(1.88$ $\mu \mathrm{g} / \mathrm{g}), \mathrm{VICH}-5(1.87 \mu \mathrm{g} / \mathrm{g})$ were noticed to have Cry1Ac protein in seed significantly higher than critical level $(1.90 \mu \mathrm{g} / \mathrm{g})$ at 120 DAS. The Bt hybrids viz., JKCH-2245 (1.87 $\mu \mathrm{g} / \mathrm{g}), \mathrm{JKCH}-22(2.59 \mu \mathrm{g} / \mathrm{g}), \mathrm{SBCH}-311$ (2.87 $\mu \mathrm{g} / \mathrm{g})$, Dhruva $(2.34 \mu \mathrm{g} / \mathrm{g})$, JK-Varun $(2.21 \mu \mathrm{g} / \mathrm{g})$ and JK-Durga $(2.61 \mu \mathrm{g} / \mathrm{g})$ showed Cry1Ac protein more than critical level at 160 DAS in seed tissue.Kranthiet al., (2005) reported $1.77+0.23 \mu \mathrm{g}$ per g Cry1Ac protein content in seeds. The Cry1Ac endotoxin level in flower at different stages of the crop has been presented in Table 4 and Figure 4.

None of the Bt hybrids had significantly higher Cry1Ac protein than critical level required for mortality $(1.90 \mu \mathrm{g} / \mathrm{g})$ of pink boll worm at 105 and 140 DAS in the flower. But, at 120 DAS, hybrids JKCH-1947 (2.36 $\mu \mathrm{g} / \mathrm{g})$, RCH-134 (2.36 $\mu \mathrm{g} / \mathrm{g})$, JKCH-22 $(2.11 \mu \mathrm{g} / \mathrm{g})$, Ankur-651 (3.25 $\mu \mathrm{g} / \mathrm{g})$ and JKCH-29 (2.21 $\mu \mathrm{g} / \mathrm{g})$ recorded Cry1Ac protein significantly higher than critical level $(1.90 \mu \mathrm{g} / \mathrm{g})$. There was a decrease in the Cry1Ac protein level from 120 to 140 DAS. Rest of the hybrids exhibited Cry1Ac protein lesser $(1.20 \mu \mathrm{g} / \mathrm{g})$ than critical level $(1.90 \mu \mathrm{g} / \mathrm{g})$ in flower.

The estimated quantity of Cry1Ac protein in young bolls is presented in Table 5 and Figure
5. The Cry1Ac expression at 105, 120 and 140 DAS in young boll was $2.20,0.96$ and $0.32 \mu \mathrm{g}$ per g. The per cent decrease of Cry1Ac protein from 105 to 120 DAS was 56.36. From 120 to 140 DAS, decrease of Cry1Ac protein was 66.67 per cent.

The hybrids viz., JKCH-2245 (1.88 $\mu \mathrm{g} / \mathrm{g})$, SBCH-302 $(2.44 \mu \mathrm{g} / \mathrm{g}), \mathrm{RCH}-2(2.40 \mu \mathrm{g} / \mathrm{g})$, JK-Ishwar $(2.49 \mu \mathrm{g} / \mathrm{g}), \quad \mathrm{JKCH}-1947 \quad(4.29$ $\mu \mathrm{g} / \mathrm{g}), \mathrm{RCH}-134$ (2.61 $\mu \mathrm{g} / \mathrm{g}), \mathrm{JKCH}-22$ (2.07 $\mu \mathrm{g} / \mathrm{g})$, Ankur-651 (1.98 $\mu \mathrm{g} / \mathrm{g}), \mathrm{RCH}-144$ (2.85 $\mu \mathrm{g} / \mathrm{g}), \mathrm{SBCH}-311(2.11 \mu \mathrm{g} / \mathrm{g}), \mathrm{PCH}-2270$ (1.90 $\mu \mathrm{g} / \mathrm{g}), \mathrm{NCEN}-3 \mathrm{R}(3.33 \mu \mathrm{g} / \mathrm{g}), \mathrm{KDCHH}-9810$ (2.66 $\mu \mathrm{g} / \mathrm{g})$, Dhruva $(2.02 \mu \mathrm{g} / \mathrm{g})$, KDCHH$9632(2.74 \mu \mathrm{g} / \mathrm{g})$, JK-Varun (4.41 $\mu \mathrm{g} / \mathrm{g})$, RCH-2171 (1.97 $\mu \mathrm{g} / \mathrm{g})$, JK-Durga $(4.11 \mu \mathrm{g} / \mathrm{g})$, JKCH-99 $(2.89 \mu \mathrm{g} / \mathrm{g})$, RCH-138 (1.98 $\mu \mathrm{g} / \mathrm{g})$, JKCH-1945 (4.32 $\mu \mathrm{g} / \mathrm{g}), \quad \mathrm{RCH}-377 \quad(2.71$ $\mu \mathrm{g} / \mathrm{g}), \mathrm{VICH}-111(2.76 \mu \mathrm{g} / \mathrm{g}), \mathrm{VICH}-5$ (2.20 $\mu \mathrm{g} / \mathrm{g})$ and VICH-9 $(2.88 \mu \mathrm{g} / \mathrm{g})$ showed significantly higher Cry1Ac protein than the critical level $(1.90 \mu \mathrm{g} / \mathrm{g})$ at $105 \mathrm{DAS}$, while at 120 DAS, JKCH-2245 (2.39 $\mu \mathrm{g} / \mathrm{g})$, JKCH-99 $(3.46 \mu \mathrm{g} / \mathrm{g})$ and $\mathrm{K}-5038(2.85 \mu \mathrm{g} / \mathrm{g})$ only had Cry1Ac protein significantly higher than critical level in young boll. All 36 hybrids produced Cry1Ac protein lesser than critical level $(1.90 \mu \mathrm{g} / \mathrm{g})$ in young boll at 140 DAS.

\section{Summary}

In leaf tissue all tested hybrids produced higher Cry 1Ac protein than critical level $(1.9 \mu \mathrm{g} / \mathrm{g})$ except JKCH-1050 and JKCH-226 at the stage of 45 DAS. After 90 days Cry 1Ac protein more than critical level especially in JKCH-2245, SBCH-302, K-5308, KDCHH441, JK-Indra, RCH-2, JK-Iswar, JKCH-1947, RCH-134, JKCH-22, Ankur-651, RCH-144, NCEN-3R, KDCHH-9810, Druva, RCH-2171, RCH-20, RCH-138，JKCH-1945， RCH-377 and $\mathrm{NHH}-44$ but none of Bt hybrid exhibited more than critical level $(1.9 \mu \mathrm{g} / \mathrm{g})$ at the stage of 120DAS, 140 DAS and 160 DAS. 
Table.1 Cry1Ac endotoxin $(\mu \mathrm{g} / \mathrm{g})$ content in leaf at different stages of crop growth of Bt cotton genotypes at Dharwad

\begin{tabular}{|c|c|c|c|c|c|c|}
\hline $\begin{array}{l}\text { Sl. } \\
\text { No. }\end{array}$ & Bt genotypes & 45 days & 90 days & 120 days & 140 days & 160 days \\
\hline 1. & JKCH-2245 & $5.27 * *$ & $2.85 * *$ & $0.64 *$ & $0.41 * *$ & $0.27 *$ \\
\hline 2. & SBCH-302 & 3.52 & 2.13 & 0.39 & $0.99 * *$ & $0.40 * *$ \\
\hline 3. & K-5308 & 3.44 & 2.02 & $0.58 * *$ & $0.88^{* *}$ & $0.47 * *$ \\
\hline 4. & KDCHH-441 & 4.02 & 2.25 & 0.46 & 0.37 & 0.17 \\
\hline 5. & JK Indra & 3.84 & 2.21 & $0.69 * *$ & $0.45^{* *}$ & 0.08 \\
\hline 6. & $\mathrm{RCH}-2$ & 4.73 & 2.79 & 0.53 & 0.37 & 0.18 \\
\hline 7. & JK Ishwar & 4.44 & 2.25 & $0.99 * *$ & $0.85^{* *}$ & $0.37 * *$ \\
\hline 8. & JKCH-1947 & $6.73 * *$ & $3.48 * *$ & $1.59 * *$ & $1.35^{* *}$ & $0.77 * *$ \\
\hline 9. & RCH-134 & $5.13 * *$ & 2.79 & $0.94 * *$ & $0.53 * *$ & $0.29 * * *$ \\
\hline 10. & JKCH-22 & $6.41 * *$ & $3.04 * *$ & $0.99 * *$ & $0.85^{* *}$ & $0.50 * * *$ \\
\hline 11. & Ankur-651 BGI & 4.70 & 1.99 & $0.57 * *$ & $0.49 * *$ & $0.33 * *$ \\
\hline 12. & RCH-144 & 4.86 & $2.95 * *$ & $0.69 * *$ & $0.51 * *$ & $0.30 * *$ \\
\hline 13. & JKCH-1050 & 0.07 & 0.09 & 0.08 & 0.06 & 0.00 \\
\hline 14. & SBCH-311 & 3.63 & 1.10 & $1.00 * *$ & $0.82 * *$ & $0.50 * * *$ \\
\hline 15. & PCH-2270 & 4.51 & 1.62 & $0.67 * *$ & $0.52 * *$ & $0.25 * *$ \\
\hline 16. & NECH-3R & 2.89 & 2.21 & $1.09 * *$ & $1.03 * *$ & $0.42 *$ \\
\hline 17. & КDCHН-9810 & 4.64 & 2.26 & $0.64 * *$ & $0.44 * *$ & $0.83 * *$ \\
\hline 18. & NCEN-2R & $4.87 * *$ & 0.03 & 0.02 & 0.04 & 0.00 \\
\hline 19. & RCH-118 & 3.52 & 1.73 & $0.90 * *$ & $0.64 * *$ & $0.39 * *$ \\
\hline 20. & JKCH-226 & 0.08 & 0.08 & 0.01 & 0.00 & 0.00 \\
\hline 21. & Dhruva & 3.60 & 2.40 & $1.15^{* *}$ & $0.89 * *$ & $0.42 * *$ \\
\hline 22. & K-5316 & 2.56 & 1.04 & $1.08^{*}$ & $0.97 * *$ & $0.44 * *$ \\
\hline 23. & KDCHH-9632 & 4.10 & 1.93 & $1.09 *$ & $0.83^{* *}$ & $0.54 * *$ \\
\hline 24. & JK Varun & $6.07 * *$ & 0.28 & $0.99 * *$ & $0.73^{* *}$ & $0.50 * *$ \\
\hline 25. & RCH-2171 & $5.49 * *$ & 2.64 & $0.98 * *$ & $0.64 * *$ & $0.30 * *$ \\
\hline 26. & RCH-20 & 4.15 & 2.23 & $0.69 * *$ & $0.43 * *$ & $0.36^{* * * *}$ \\
\hline 27. & JK Durga & $7.26^{* *}$ & 1.84 & $1.02 * *$ & $0.76^{* *}$ & $0.33 * *$ \\
\hline 28. & JKCH-99 & 3.72 & 1.84 & 0.52 & $0.53^{* *}$ & $0.33 * *$ \\
\hline 29. & RCH-138 & 3.72 & 1.95 & 0.55 & $0.44 * *$ & $0.20 * *$ \\
\hline 30. & JKCH-1945 & $5.85 * *$ & 2.20 & $0.83 * *$ & $0.77 * *$ & $0.40 * *$ \\
\hline 31. & JK Gowri & 0.03 & 0.09 & 0.01 & 0.00 & 0.00 \\
\hline 32. & RCH-377 & $5.25 * *$ & 2.39 & $1.00 * *$ & $0.75^{* *}$ & $0.55^{* * *}$ \\
\hline 33. & VICH-111 & $4.96^{* *}$ & 1.01 & 0.53 & 0.35 & $0.25 \% *$ \\
\hline 34. & NHH-44 & 4.20 & 1.90 & 0.55 & 0.31 & 0.17 \\
\hline 35. & VICH-5 & 2.71 & 0.62 & $0.80 * *$ & $0.53 * *$ & $0.36 \% *$ \\
\hline \multirow[t]{6}{*}{36.} & VICH-9 & $5.69 * *$ & 1.68 & $0.83 * *$ & $0.62 * *$ & $0.44 * *$ \\
\hline & Grand mean & 4.21 & 1.83 & 0.73 & 0.60 & 0.33 \\
\hline & $\mathrm{SEm} \pm$ & 0.035 & 0.0117 & 0.0092 & 0.0016 & 0.005 \\
\hline & $\mathrm{CD}$ at $5 \%$ & 0.10 & 0.033 & 0.026 & 0.0047 & 0.0143 \\
\hline & $\mathrm{CD}$ at $1 \%$ & 0.13 & 0.045 & 0.035 & 0.0064 & 0.0192 \\
\hline & CV (\%) & 1.19 & 0.90 & 1.792 & 0.39 & 2.13 \\
\hline
\end{tabular}

$*$, **: Significant at $5 \%$ and $1 \%$ level of probability 
Table.2 Cry1Ac endotoxin $(\mu \mathrm{g} / \mathrm{g})$ content in boll rinds at different stages of crop growth of Bt cotton genotypes at Dharwad

\begin{tabular}{|c|c|c|c|c|c|}
\hline \multirow[t]{2}{*}{ SI. No. } & \multirow[t]{2}{*}{ Bt genotypes } & \multicolumn{4}{|c|}{ Rind } \\
\hline & & 105 days & 120 days & 140 days & 160 days \\
\hline 1. & JKCH-2245 & $1.04 * *$ & 0.43 & 0.35 & 0.14 \\
\hline 2. & SBCH-302 & 0.24 & $0.57 * *$ & 0.22 & 0.07 \\
\hline 3. & K-5308 & 0.16 & 0.38 & 0.48 & $0.59 * *$ \\
\hline 4. & KDCHH-441 & 0.29 & 0.22 & 0.28 & 0.26 \\
\hline 5. & JK Indra & $0.41 * *$ & $0.56 * *$ & 0.34 & 0.26 \\
\hline 6. & $\mathrm{RCH}-2$ & 0.30 & 0.41 & 0.51 & $0.47 * * *$ \\
\hline 7. & JK Ishwar & $1.41 * *$ & 0.26 & 0.39 & 0.26 \\
\hline 8. & JKCH-1947 & $0.48 * *$ & 0.36 & 0.36 & 0.16 \\
\hline 9. & RCH-134 & $1.47 * *$ & 0.49 & 0.35 & $0.57 * *$ \\
\hline 10. & JKCH-22 & $1.37 * *$ & $0.75^{* *}$ & 0.28 & 0.17 \\
\hline 11. & Ankur-651 BGI & 0.27 & 0.16 & 0.35 & 0.12 \\
\hline 12. & RCH-144 & 0.00 & $0.71 * *$ & $0.54 * *$ & $0.53 * *$ \\
\hline 13. & JKCH-1050 & $1.11 * *$ & $0.78 * *$ & $0.60 * *$ & 0.11 \\
\hline 14. & SBCH-311 & 0.28 & $0.92 * *$ & $0.79 * *$ & $0.61^{* * *}$ \\
\hline 15. & PCH-2270 & $0.37 *$ & $0.66^{* * *}$ & 0.48 & $0.56 * *$ \\
\hline 16. & NECH-3R & $0.46 * *$ & $0.87 * *$ & $0.64 * *$ & $0.57 * *$ \\
\hline 17. & KDCHH-9810 & $0.43 * *$ & 0.33 & 0.17 & 0.03 \\
\hline 18. & NCEN-2R & $0.64 * *$ & 0.41 & 0.35 & 0.00 \\
\hline 19. & RCH-118 & $0.44 * *$ & 0.42 & 0.32 & 0.00 \\
\hline 20. & JKCH-226 & $0.37 * *$ & 0.32 & 0.08 & 0.00 \\
\hline 21. & Dhruva & $0.60 * *$ & 0.40 & 0.33 & 0.13 \\
\hline 22. & K-5316 & $0.68 * *$ & $0.67 * *$ & 0.36 & 0.23 \\
\hline 23. & KDCHH-9632 & $0.87 * *$ & $0.57 * *$ & $0.53^{*}$ & 0.32 \\
\hline 24. & JK Varun & $0.69 * *$ & $0.55^{* *}$ & 0.52 & 0.47 \\
\hline 25. & RCH-2171 & $0.71 * *$ & 0.38 & $0.71 * *$ & 0.39 \\
\hline 26. & $\mathrm{RCH}-20$ & $0.79 * *$ & $0.65^{* *}$ & 0.45 & 0.45 \\
\hline 27. & JK Durga & $1.22 * *$ & $1.00 * *$ & $0.67 * *$ & $0.56 * *$ \\
\hline 28. & JKCH-99 & $0.71 * *$ & $0.67 * *$ & $0.63 * *$ & 0.33 \\
\hline 29. & RCH-138 & $0.70 * *$ & 0.42 & 0.36 & 0.23 \\
\hline 30. & JKCH-1945 & $1.16 * *$ & $1.06 * *$ & $0.70 * *$ & 0.00 \\
\hline 31. & JK Gowri & 0.09 & 0.04 & 0.03 & 0.00 \\
\hline 32. & RCH-377 & $0.43 * *$ & 0.43 & 0.32 & 0.28 \\
\hline 33. & VICH-111 & $0.62 * *$ & 0.41 & 0.33 & 0.16 \\
\hline 34. & NHH-44 & $0.71 * *$ & $0.57 * *$ & 0.40 & 0.25 \\
\hline 35. & VICH-5 & $0.72 * *$ & $0.57 * *$ & 0.42 & 0.28 \\
\hline 36. & VICH-9 & $0.43 * *$ & $0.50 * *$ & 0.32 & 0.23 \\
\hline & Grand mean & 0.63 & 0.53 & 0.43 & 0.27 \\
\hline & SEm \pm & 0.008 & 0.0129 & 0.0059 & 0.008 \\
\hline & $\mathrm{CD}$ at $5 \%$ & 0.025 & 0.037 & 0.017 & 0.023 \\
\hline & $\mathrm{CD}$ at $1 \%$ & 0.033 & 0.049 & 0.022 & 0.032 \\
\hline & CV (\%) & 1.92 & 3.49 & 1.93 & 4.32 \\
\hline
\end{tabular}

*,**: Significant at $5 \%$ and $1 \%$ level of probability 
Table.3 Cry1 Ac endotoxin $(\mu \mathrm{g} / \mathrm{g})$ content in seeds at different stages of crop growth of Bt cotton genotypes at Dharwad

\begin{tabular}{|c|c|c|c|c|c|}
\hline \multirow{2}{*}{$\begin{array}{l}\text { SI. } \\
\text { No. }\end{array}$} & \multirow[t]{2}{*}{ Bt genotypes } & \multicolumn{4}{|c|}{ Seeds } \\
\hline & & 105 days & 120 days & 140 days & 160 days \\
\hline 1. & JKCH-2245 & 0.68 & $1.87 * *$ & $0.62 *$ & $1.87 * *$ \\
\hline 2. & SBCH-302 & 0.74 & $2.33 * *$ & $0.78 * *$ & 1.27 \\
\hline 3. & K-5308 & $0.85^{* *}$ & $2.58 * *$ & $0.86 * *$ & 1.59 \\
\hline 4. & KDCHH-441 & 0.68 & 1.74 & 0.58 & 1.59 \\
\hline 5. & JK Indra & 0.70 & 1.22 & 0.41 & 1.14 \\
\hline 6. & $\mathrm{RCH}-2$ & 0.71 & 1.78 & 0.59 & 1.58 \\
\hline 7. & JK Ishwar & 0.47 & 1.30 & 0.43 & 0.74 \\
\hline 8. & JKCH-1947 & $1.10 * *$ & $3.22 * *$ & $1.07 * *$ & $1.07 \% *$ \\
\hline 9. & RCH-134 & 0.71 & 1.79 & 0.60 & $1.71 * *$ \\
\hline 10. & JKCH-22 & $1.13 * *$ & $3.35^{* *}$ & $1.12 * *$ & 2.59 \\
\hline 11. & Ankur-651 BGI & 0.69 & 1.53 & 0.51 & $1.15 * *$ \\
\hline 12. & RCH-144 & 0.68 & $1.84 * *$ & 0.61 & 1.71 \\
\hline 13. & JKCH-1050 & 0.17 & 0.12 & 0.09 & 0.09 \\
\hline 14. & SBCH-311 & $1.61 * *$ & $4.15^{* *}$ & $1.38 * *$ & $2.87 * * 6$ \\
\hline 15. & PCH-2270 & 1.08 & $2.98 * *$ & $0.99 * *$ & $2.81 * *$ \\
\hline 16. & NECH-3R & 0.71 & 1.66 & 0.55 & $1.66 * * 6$ \\
\hline 17. & KDCHH-9810 & 0.70 & $1.92 * *$ & $0.64 * *$ & $1.81 * *$ \\
\hline 18. & NCEN-2R & 0.10 & 0.27 & 0.09 & 0.24 \\
\hline 19. & RCH-118 & 0.68 & $1.84 * *$ & 0.61 & $1.69 * *$ \\
\hline 20. & JKCH-226 & 0.25 & 0.45 & 0.15 & 0.34 \\
\hline 21. & Dhruva & $1.41 * *$ & $4.17 * *$ & $1.39 * *$ & $2.34 * *$ \\
\hline 22. & K-5316 & $0.75^{*}$ & $2.20 * *$ & $0.73 * *$ & $1.68 * *$ \\
\hline 23. & KDCHH-9632 & $0.94 * *$ & $2.28 * *$ & $0.76^{* *}$ & $1.68 * * *$ \\
\hline 24. & JK Varun & $0.81 * *$ & $2.33 * *$ & $0.78 * *$ & $2.21^{* * *}$ \\
\hline 25. & RCH-2171 & 0.58 & 1.75 & 0.58 & $1.64 *$ \\
\hline 26. & RCH-20 & 0.67 & $1.87 * *$ & $0.62 *$ & 1.73 \\
\hline 27. & JK Durga & $1.03 * *$ & $3.01 * *$ & $1.00 * *$ & $2.61 * * 6$ \\
\hline 28. & JKCH-99 & 0.67 & 1.32 & 0.44 & 1.14 \\
\hline 29. & RCH-138 & 0.69 & 1.32 & 0.44 & 1.1 \\
\hline 30. & JKCH-1945 & $1.03 * *$ & $2.11 * *$ & $0.70 * *$ & 1.42 \\
\hline 31. & JK Gowri & 0.14 & 0.34 & 0.11 & 0.33 \\
\hline 32. & RCH-377 & 0.68 & 1.23 & 0.41 & 0.91 \\
\hline 33. & VICH-111 & 0.71 & $1.88 * *$ & $0.63 * *$ & $1.83 * *$ \\
\hline 34. & NHH-44 & 0.68 & $1.78 * *$ & 0.59 & 1.25 \\
\hline 35. & VICH-5 & 0.68 & $1.87 * *$ & $0.62 *$ & 1.32 \\
\hline 36. & VICH-9 & 0.34 & 1.09 & 0.36 & 1.07 \\
\hline & Grand mean & 0.73 & 1.90 & 0.64 & 1.49 \\
\hline & $\mathrm{SEm} \pm$ & 0.0127 & 0.013 & 0.0098 & 0.0072 \\
\hline & $\mathrm{CD}$ at $5 \%$ & 0.036 & 0.039 & 0.028 & 0.020 \\
\hline & $\mathrm{CD}$ at $1 \%$ & 0.049 & 0.059 & 0.037 & 0.027 \\
\hline & CV (\%) & 2.46 & 1.01 & 2.16 & 0.68 \\
\hline
\end{tabular}

*, **: Significant at $5 \%$ and $1 \%$ level of probability 
Table.4 Cry1Ac endotoxin $(\mu \mathrm{g} / \mathrm{g})$ content in flower at different stages of crop growth of $\mathrm{Bt}$ cotton genotypes at Dharwad

\begin{tabular}{|c|c|c|c|c|}
\hline \multirow[t]{2}{*}{ Sl. No. } & \multirow[t]{2}{*}{ Bt genotypes } & \multicolumn{3}{|c|}{ Flower } \\
\hline & & 105 days & 120 days & 140 days \\
\hline 1. & JKCH-2245 & 0.07 & 0.26 & 0.09 \\
\hline 2. & SBCH-302 & 0.23 & 0.18 & 0.06 \\
\hline 3. & K-5308 & 0.26 & 0.13 & 0.04 \\
\hline 4. & KDCHH-441 & 0.10 & 0.17 & 0.06 \\
\hline 5. & JK Indra & 0.12 & 0.04 & 0.01 \\
\hline 6. & $\mathrm{RCH}-2$ & 0.31 & 0.46 & 0.15 \\
\hline 7. & JK Ishwar & 0.09 & 0.13 & 0.04 \\
\hline 8. & JKCH-1947 & $0.88 * *$ & $2.36 * *$ & $0.79 \div \div$ \\
\hline 9. & RCH-134 & $0.92 * *$ & $2.36 * *$ & $0.70 \% *$ \\
\hline 10. & JKCH-22 & $1.29 * *$ & $2.11 * *$ & $1.12 * *$ \\
\hline 11. & Ankur-651 BGI & 0.16 & $3.25 * *$ & 0.11 \\
\hline 12. & RCH-144 & $0.40 * *$ & $0.33^{*}$ & $0.37 * * 6$ \\
\hline 13. & JKCH-1050 & 0.24 & $0.59 * *$ & $0.20 * *$ \\
\hline 14. & SBCH-311 & $0.43 * *$ & $0.90 * *$ & $0.30 * *$ \\
\hline 15. & PCH-2270 & 0.27 & $0.59 * *$ & $0.20 *$ \\
\hline 16. & NECH-3R & 0.27 & 0.31 & 0.10 \\
\hline 17. & KDCHH-9810 & 0.18 & 0.22 & 0.07 \\
\hline 18. & NCEN-2R & 0.23 & 0.42 & 0.14 \\
\hline 19. & RCH-118 & 0.11 & 0.13 & 0.04 \\
\hline 20. & JKCH-226 & $0.42 * *$ & $0.96 * *$ & $0.32 * *$ \\
\hline 21. & Dhruva & $0.60 * *$ & $1.33 * *$ & $0.44 * *$ \\
\hline 22. & K-5316 & 0.23 & 0.25 & 0.08 \\
\hline 23. & KDCHH-9632 & 0.11 & 0.19 & 0.06 \\
\hline 24. & JK Varun & $0.57 * *$ & $1.12 * *$ & $0.37 * *$ \\
\hline 25. & RCH-2171 & 0.10 & 0.41 & 0.14 \\
\hline 26. & $\mathrm{RCH}-20$ & 0.24 & 0.25 & 0.08 \\
\hline 27. & JK Durga & 0.18 & 0.21 & 0.07 \\
\hline 28. & JKCH-99 & $1.08 * *$ & $2.21 * *$ & $0.74 * *$ \\
\hline 29. & RCH-138 & 0.26 & $0.64 * *$ & $0.21 * *$ \\
\hline 30. & JKCH-1945 & $0.58 * *$ & $1.19 * *$ & $0.40 \div \%$ \\
\hline 31. & JK Gowri & 0.24 & 0.32 & 0.11 \\
\hline 32. & RCH-377 & $0.42 * *$ & $0.91 * *$ & $0.30 \div \div$ \\
\hline 33. & VICH-111 & 0.27 & $0.53 * *$ & $0.18 * *$ \\
\hline 34. & NHH-44 & 0.24 & 0.32 & 0.11 \\
\hline 35. & VICH-5 & 0.18 & 0.26 & 0.09 \\
\hline \multirow[t]{6}{*}{36.} & VICH-9 & $0.45 * *$ & $1.14 * *$ & $0.38 * *$ \\
\hline & Grand mean & 0.35 & 0.72 & 0.24 \\
\hline & SEm \pm & 0.0042 & 0.011 & 0.0074 \\
\hline & $\mathrm{CD}$ at $5 \%$ & 0.012 & 0.034 & 0.021 \\
\hline & $\mathrm{CD}$ at $1 \%$ & 0.016 & 0.046 & 0.028 \\
\hline & CV (\%) & 1.6975 & 2.34 & 4.39 \\
\hline
\end{tabular}

$*$,**: Significant at $5 \%$ and $1 \%$ level of probability 
Table.5 Cry1Ac endotoxin $(\mu \mathrm{g} / \mathrm{g})$ content in young boll at different stages of crop growth of Bt cotton genotypes at Dharwad

\begin{tabular}{|c|c|c|c|c|}
\hline \multirow{2}{*}{ SI. No. } & \multirow[t]{2}{*}{ Bt genotypes } & \multicolumn{3}{|c|}{ Young boll } \\
\hline & & 105 days & 120 days & 140 days \\
\hline 1. & JKCH-2245 & 1.88 & $2.39 * *$ & 0.80 \\
\hline 2. & SBCH-302 & 2.44 & 0.76 & 0.25 \\
\hline 3. & K-5308 & 0.98 & $2.85 * *$ & $0.95 * *$ \\
\hline 4. & KDCHH-441 & 0.88 & 0.78 & 0.26 \\
\hline 5. & JK Indra & 1.44 & 0.82 & 0.27 \\
\hline 6. & $\mathrm{RCH}-2$ & 2.46 & 0.83 & 0.28 \\
\hline 7. & JK Ishwar & 2.49 & 0.87 & 0.29 \\
\hline 8. & JKCH-1947 & $4.29 * *$ & $0.94 * *$ & $0.31 *$ \\
\hline 9. & RCH-134 & $2.61 * *$ & 0.75 & 0.25 \\
\hline 10. & JKCH-22 & 2.07 & 0.72 & 0.24 \\
\hline 11. & Ankur-651 BGI & 1.98 & 0.81 & 0.27 \\
\hline 12. & RCH-144 & $2.85^{* *}$ & 0.65 & 0.22 \\
\hline 13. & JKCH-1050 & 0.97 & 0.6 & 0.20 \\
\hline 14. & SBCH-311 & 2.11 & 0.56 & 0.19 \\
\hline 15. & PCH-2270 & 1.92 & 0.76 & 0.25 \\
\hline 16. & NECH-3R & $3.33 * *$ & 0.78 & 0.26 \\
\hline 17. & KDCHH-9810 & $2.66 * *$ & 0.71 & 0.24 \\
\hline 18. & NCEN-2R & 1.22 & 0.59 & 0.20 \\
\hline 19. & RCH-118 & 1.28 & $0.92 * *$ & $0.31 *$ \\
\hline 20. & JKCH-226 & 0.93 & 0.73 & 0.24 \\
\hline 21. & Dhruva & 2.02 & 0.68 & 0.23 \\
\hline 22. & K-5316 & 1.01 & 0.7 & 0.23 \\
\hline 23. & KDCHH-9632 & $2.74 * *$ & 1.26 & $0.42 * \%$ \\
\hline 24. & JK Varun & $4.41 * *$ & 0.81 & 0.27 \\
\hline 25. & RCH-2171 & 1.97 & 0.66 & 0.22 \\
\hline 26. & RCH-20 & 1.56 & 0.78 & 0.26 \\
\hline 27. & JK Durga & $4.11 * *$ & $0.88 * *$ & 0.29 \\
\hline 28. & JKCH-99 & $2.82 * *$ & $3.46 * *$ & $1.15 * *$ \\
\hline 29. & RCH-138 & 1.98 & 0.54 & 0.18 \\
\hline 30. & JKCH-1945 & $4.32 * *$ & 0.72 & 0.24 \\
\hline 31. & JK Gowri & 0.86 & 0.83 & 0.28 \\
\hline 32. & RCH-377 & $2.71 * *$ & 0.56 & 0.19 \\
\hline 33. & VICH-111 & $2.76 * *$ & $1.05 * *$ & $0.35 * *$ \\
\hline 34. & NHH-44 & 1.23 & 0.87 & 0.29 \\
\hline 35. & VICH-5 & 2.12 & $1.01 * *$ & $0.34 * *$ \\
\hline \multirow[t]{6}{*}{36.} & VICH-9 & $2.88 * *$ & 0.85 & 0.28 \\
\hline & Grand mean & 2.20 & 0.96 & 0.32 \\
\hline & $\mathrm{SEm} \pm$ & 0.02 & 0.0146 & 0.01 \\
\hline & $\mathrm{CD}$ at $5 \%$ & 0.057 & 0.042 & 0.029 \\
\hline & $\mathrm{CD}$ at $1 \%$ & 0.077 & 0.05 & 0.0389 \\
\hline & CV (\%) & 1.29 & 2.16 & 4.48 \\
\hline
\end{tabular}

$*, * *$ Significant at $5 \%$ and $1 \%$ level of probability 
Table.6 Crop growth stage-wise expression trend of Cry1Ac protein in different tissues of cotton at Dharwad

\begin{tabular}{|c|c|c|c|c|c|c|c|c|c|c|}
\hline & & & & & Cry1Ac pl & $\operatorname{tein}(\mu g / g)$ & & & & \\
\hline & 45 DAS & 90 DAS & $\begin{array}{r}\% \text { over } \\
45 \text { DAS }\end{array}$ & 105 DAS & 120 DAS & $\begin{array}{c}\% \text { over } \\
105 \text { DAS }\end{array}$ & 140 DAS & $\begin{array}{c}\% \text { over } \\
120 \text { DAS }\end{array}$ & 160 DAS & $\begin{array}{c}\% \text { over } \\
140 \\
\text { DAS }\end{array}$ \\
\hline Leaf & 4.21 & 1.83 & -56.54 & - & 0.73 & $-60.11^{+}$ & 0.60 & -17.82 & 0.33 & -45.00 \\
\hline Rind & - & - & & 0.63 & 0.53 & -15.88 & 0.43 & -18.87 & 0.23 & -46.51 \\
\hline Seed & - & - & & 0.73 & 1.90 & 160.27 & 0.64 & -296.88 & 1.49 & 232.81 \\
\hline $\begin{array}{l}\text { Young } \\
\text { boll }\end{array}$ & - & - & & 2.20 & 0.96 & -56.36 & 0.32 & -66.67 & - & \\
\hline Flower & - & - & & 0.35 & 0.72 & 105.71 & 0.24 & -66.67 & - & \\
\hline
\end{tabular}

$+\%$ over 90 DAS

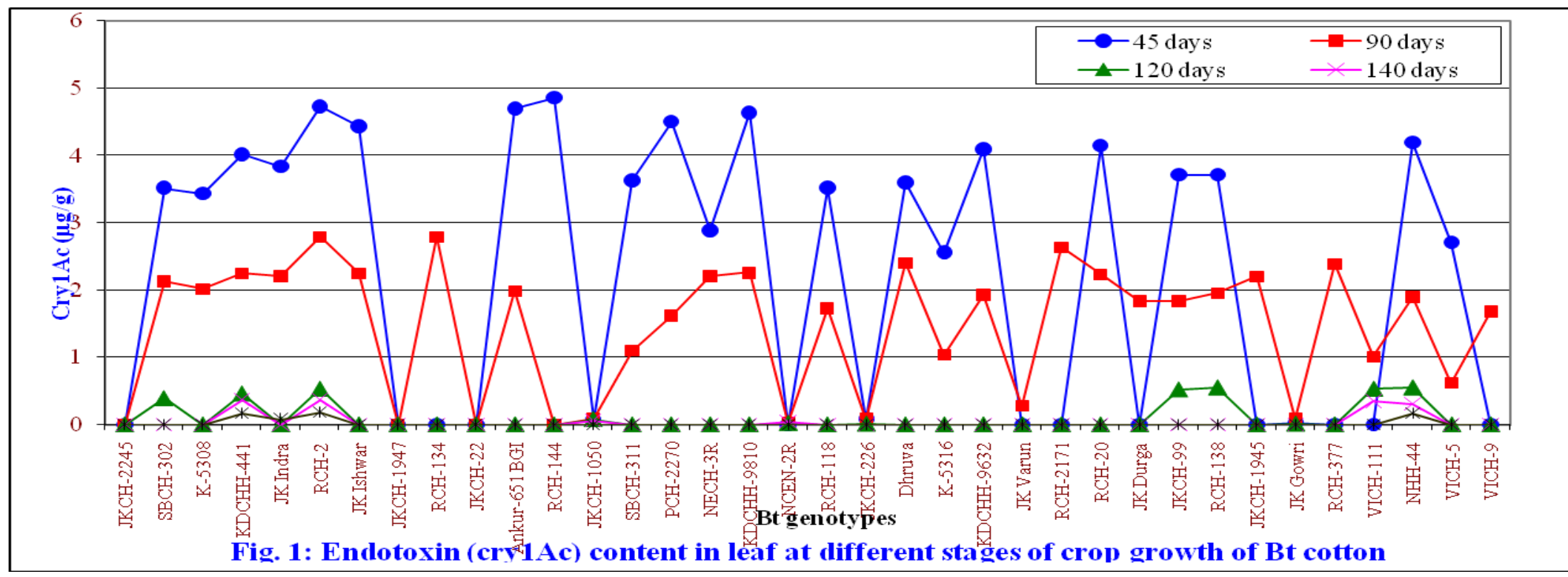



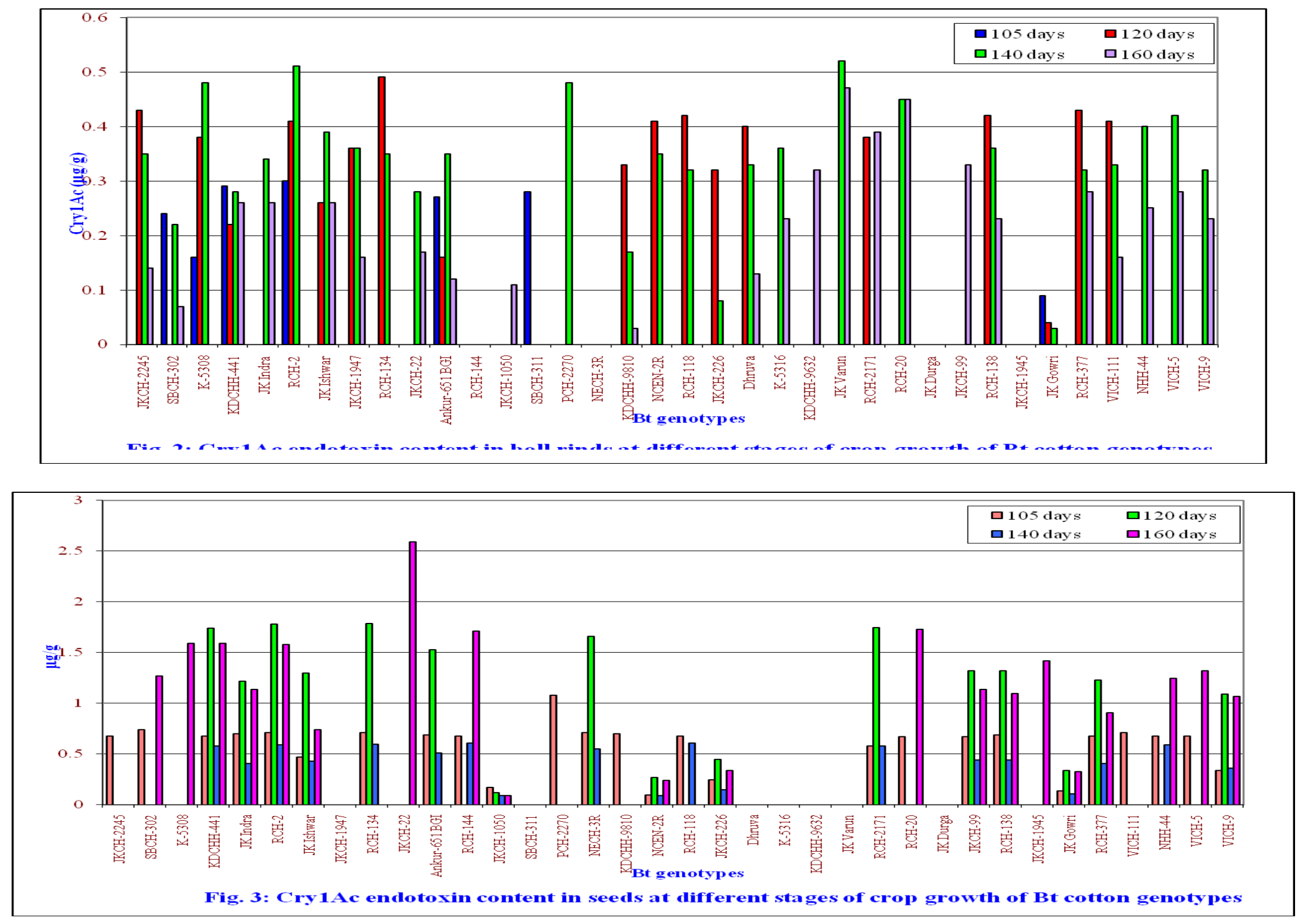

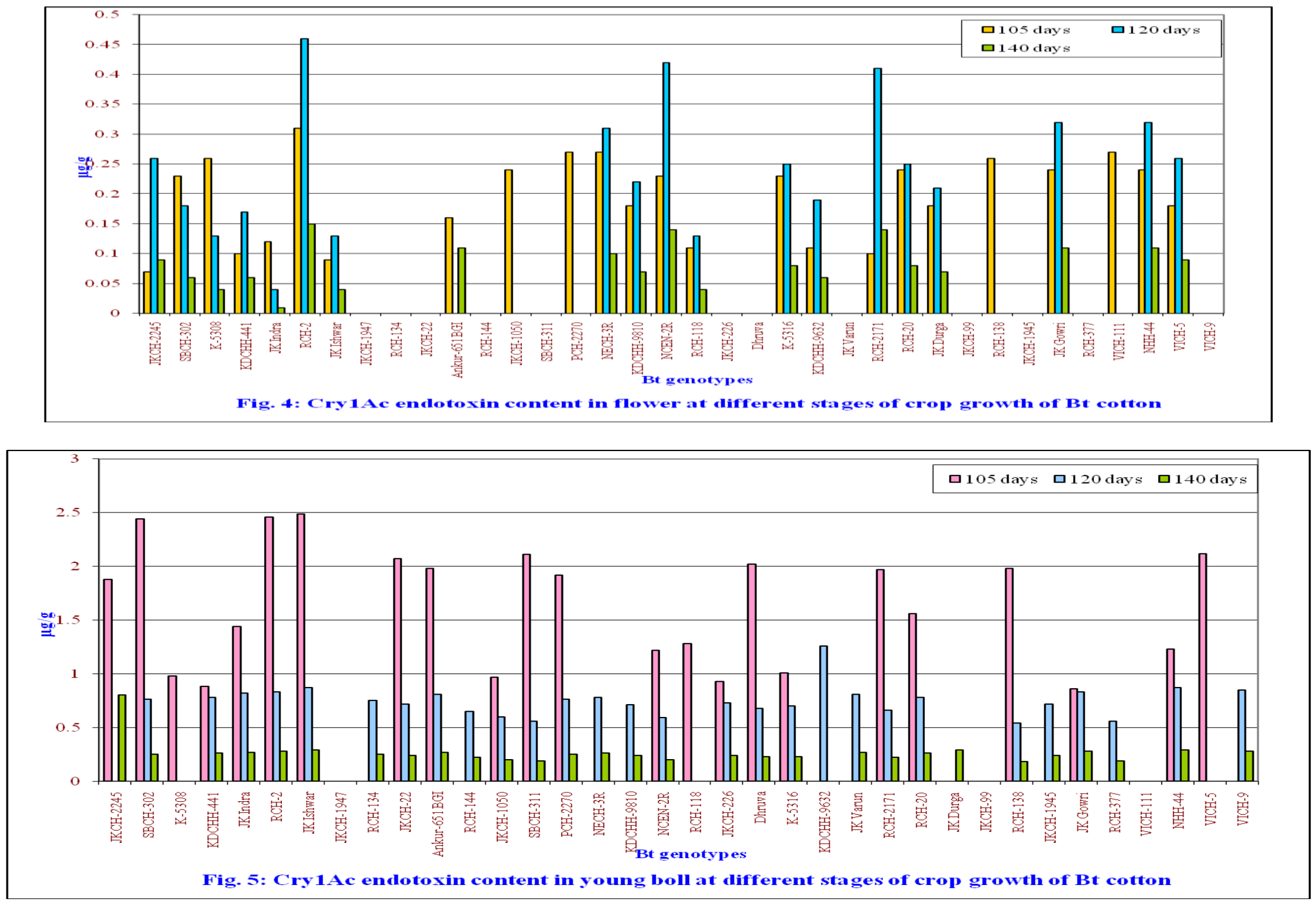
JKCH-1947 produced Cry 1 AC protein highest at 120 DAS $(1.59 \mu \mathrm{g} / \mathrm{g}), 140$ DAS $(1.35 \mu \mathrm{g} / \mathrm{g})$ and 160 DAS $(0.77 \mu \mathrm{g} / \mathrm{g})$.

In young boll highest $\mathrm{Bt}$ protein produced at 105 DAS followed by 120 and 140 DAS. At the stage of 105 DAS, most of Bt hybrids produced Cry 1Ac protein more than critical level except K-5308, KDCHH-441, JK-Indra, JKCH1050, NCEN-2R, RCH-18, JKCH226,K-5316, RCH-20, JK-Gouri and NHH44. K-5308 (2.85(1.29 $\mu \mathrm{g} / \mathrm{g})$, JKCH-99 (3.46) and JKCH-2245 (2.39) exhibited Bt protein more than critical level after 120 days after sowing. None of $\mathrm{Bt}$ hybrid produced endotoxin more than critical level at the stage of 140 DAS while, JKCH-99 $(1.15 \mu \mathrm{g} / \mathrm{g})$ noticed highest Bt protein.

In seed tissue, SBCH-302 $(2.33 \mu \mathrm{g} / \mathrm{g}), \mathrm{K}$ $5308(2.58 \mu \mathrm{g} / \mathrm{g}), \mathrm{JKCH}-1947(3.22 \mu \mathrm{g} / \mathrm{g})$, JKCH-22 $(3.35 \mu \mathrm{g} / \mathrm{g}), \mathrm{SBCH}-311(4.15 \mu \mathrm{g} / \mathrm{g})$, PCH-2270 $(2.98 \mu \mathrm{g} / \mathrm{g}), \mathrm{KDCHH}-9810(1.92$ $\mu \mathrm{g} / \mathrm{g})$, Dhruv $(4.17 \mu \mathrm{g} / \mathrm{g}), \mathrm{K}-5316(2.2 \mu \mathrm{g} / \mathrm{g})$, KDCHH-9632 $(2.28 \mu \mathrm{g} / \mathrm{g}))$, JK-Varun (2.33 $\mu \mathrm{g} / \mathrm{g})$, JK-Durga (3.01 $\mu \mathrm{g} / \mathrm{g}), \quad \mathrm{JKCH}-1945$ $(2.11 \mu \mathrm{g} / \mathrm{g})$ produced Cry $1 \mathrm{Ac}$ protein more than critical level at 120 days after sowing. After 120 days Bt protein production was decrease at 140 DAS then Bt gene expression was increase at the stage of 160 DAS while, some of Bt hybrids viz., JKCH-22 (2.59 $\mu \mathrm{g} / \mathrm{g})$, Dhruva $(2.34 \mu \mathrm{g} / \mathrm{g})$, JK-Varun (2.21 $\mu \mathrm{g} / \mathrm{g})$ and JK-Durga $(2.61 \mu \mathrm{g} / \mathrm{g})$ noticed more than critical level but none of Bt hybrid produced Cry 1Ac protein more than critical level $(1.9 \mu \mathrm{g} / \mathrm{g})$. Out of thirty six Bt hybrid none of Bt hybrid produced endotoxin more than critical level. SBCH-311 produced highest Bt protein at the stage of 105 DAS $(1.61 \mu \mathrm{g} / \mathrm{g}), 140$ DAS $(1.38 \mu \mathrm{g} / \mathrm{g})$ and 160 DAS $(0.77 \mu \mathrm{g} / \mathrm{g})$ in seed tissue.

Average Bt protein production was maximum at the stag of 120 DAS in flower part. During this stage JKCH -1947 (2.36 $\mu \mathrm{g} / \mathrm{g})$, JKCH$134(2.36 \mu \mathrm{g} / \mathrm{g}), \mathrm{JKCH}-22(2.11 \mu \mathrm{g} / \mathrm{g}))$, Ankur $651(3.25 \mu \mathrm{g} / \mathrm{g})$ and JKCH-99 $(2.21 \mu \mathrm{g} / \mathrm{g})$ exhibited endotoxin more than critical level $(1.9 \mu \mathrm{g} / \mathrm{g})$ at 120 DAS but none of Bt hybrid recorded more than critical level of Cry $1 \mathrm{Ac}$ protein at the stage of 105 and 140 DAS. $\mathrm{JKCH}-22$ produced highest Bt protein at 105 DAS $(1.29 \mu \mathrm{g} / \mathrm{g})$ and 140 DAS $(1.12 \mu \mathrm{g} / \mathrm{g})$.

Cry 1Ac protein in rind was less than critical levelin crop life span (at 105,120 and 140 DAS). RCH-134 (1.47 $\mu \mathrm{g} / \mathrm{g}), \quad \mathrm{SBCH}-311$ $(0.79 \mu \mathrm{g} / \mathrm{g})$ and $\mathrm{K}-5308(0.59 \mu \mathrm{g} / \mathrm{g})$ exhibited highest endotoxin produced at 105 DAS, 120DAS and 140 DAS, respectively.

In conclusion, the growth stage-wise expression trend of $\mathrm{Cry} 1 \mathrm{Ac}$ protein in different tissues has been presented in Table 6 and The expression of Cry 1Ac gene highest in leaf $(4.31 \mu \mathrm{g} / \mathrm{g}$ at 45 DAS $)$ followed by young boll (2.02 $\mu \mathrm{g} / \mathrm{g}$ at $105 \mathrm{DAS})$, seeds (1.9 $\mu \mathrm{g} / \mathrm{g} 120 \mathrm{DAS}$ and $1.49 \mu \mathrm{g} / \mathrm{g}$ at $160 \mathrm{DAS})$, flower (0.72 at $120 \mathrm{DAS})$ and least in rind $(0.63 \mu \mathrm{g} / \mathrm{g} 105 \mathrm{DAS})$. Especially in leaf (45 DAS), flower (120 DAS), rind (105 DAS) and young boll (105 DAS) expression of cry 1Ac gene highest in one stage of crop life span then it decreases towards end of life (up to 160 DAS ), but in case of seed tissue Cry 1 Ac production increase from $0.73 \mu \mathrm{g} / \mathrm{g}(105$ DAS) to 1.9 (120DAS) then production was decrease to $0.64 \mu \mathrm{g} / \mathrm{g}$ (140 DAS) again it increase to1.49 $\mu \mathrm{g} / \mathrm{g}$ (160 DAS). So, expression of Cry Ac gene was varies with different plant parts.

\section{References}

Dong, H. Z. and Lis, W. J., 2007, Variability of endotoxin expression in Bt transgenic cotton J. Agron. And Crop Sci., 193(3): 21-29.

Kranthi, K. R., Naidu, S., Dhawad, C. S., 
Tatwawadi, A. M., Mate, K., Patil, E., Bharose, A. A., Behere, G. T., Wadaskar, R. M. and Kranthi, S., 2005, Temporal and intra-plant variability of cry1Ac expression by Bt cotton and its influence on the survival of the cotton bollworm, Helicoverpa armigera (Hubner) (Noctuidae : Lepidoptera). Curr. Sci., 89(2): 291-298.

\section{How to cite this article:}

Nagappa, B.H. and Khadi, B.M. 2018. Expression of Bt Gene (cry1Ac) on Different Plant Parts at Different Stages in Bt Cotton Genotypes. Int.J.Curr.Microbiol.App.Sci. 7(01): 3339-3353. doi: https://doi.org/10.20546/ijcmas.2018.701.396 